\title{
Exosomes from human induced pluripotent stem cells-derived keratinocytes accelerate burn wound healing through miR-762 mediated promotion of keratinocytes and endothelial cells migration
}

\section{Yunyao Bo}

Southern Medical University

Lijun Yang

Southern Medical University

Baiting Liu

Southern Medical University

Guiping Tian

Southern Medical University

Chenxi Li

Southern Medical University

Lin Zhang

Southern Medical University

Yuan Yan ( $\nabla$ yyuanmm@163.com)

Southern Medical University

\section{Research Article}

Keywords: Exosome, Burn, Induced pluripotent stem cells, Keratinocytes, Endothelial cell

Posted Date: May 4th, 2022

DOI: https://doi.org/10.21203/rs.3.rs-1289192/v2

License: (a) (i) This work is licensed under a Creative Commons Attribution 4.0 International License. Read Full License 


\section{Abstract \\ Background}

The use of keratinocytes derived from induced pluripotent stem cells (iPSCs-KCs) may represent a novel cell therapy strategy for burn treatment. There is growing evidence that extracellular vesicles, including exosomes, are primary mediators of the benefits of stem cell therapy. Herein, we thus explored the effects of exosomes produced by iPSCs-derived keratinocytes (iPSCs-KCs-Exos) in a model of deep seconddegree burn wound healing and evaluated the mechanistic basis for the observed activity.

\section{Methods}

iPSCs-KCs-Exos were isolated from conditioned medium of iPSCs-KCs and verified by electron micrograph and size distribution. Next, iPSCs-KCs-Exos were injected subcutaneously around wound sites, and its efficacy was evaluated by measuring wound closure areas, histological examination and immunohistochemistry staining. The effects of iPSCs-KCs-Exos on proliferation and migration of keratinocytes and endothelial cells in vitro were assessed by EdU staining, wound healing assays and transwell assay. Then, high-throughput microRNA sequencing was used to explore the underlying mechanisms. We assessed the roles of miR-762 in iPSCs-KCs-Exos-induced regulation of keratinocytes and endothelial cells migration. Furthermore, the target gene which mediated the biological effects of miR-762 in keratinocytes and endothelial cells was also been detected.

\section{Results}

The analysis revealed that iPSCs-KCs-Exos application to the burn wound drove the acceleration of wound closure, with more robust angiogenesis and re-epithelialization being evident. Such iPSCs-KCsExos treatment effectively enhanced endothelial cell and keratinocyte migration in vitro. Moreover, the enrichment of miR-762 was detected in iPSCs-KCs-Exos and was found to target promyelocytic leukemia (PML) as a means of regulating cell migration through a mechanism tie to integrin beta1 (ITGB1).

\section{Conclusion}

These results thus provide a foundation for the further study of iPSCs-KCs-Exos as novel cell-free treatments for deep second-degree burns.

\section{Introduction}

Burns are a form of serious traumatic injury that cause substantial global morbidity, and there is thus an urgent need to define novel approaches to expediting burn healing [1-3]. Preclinical and clinical evidence suggests that keratinocyte-based therapies offer great promise in the context of burn management [4-6]. 
However, there are substantial challenges to large-scale keratinocyte preparation efforts owing to the fact that donor keratinocyte yields are often very limited. The ability of these donated cells to proliferate and differentiate in vitro is also highly variable. The Yamananka lab first developed induced pluripotent stem cells (iPSCs) and highlighted their potential as a novel source of many cellular lineages for use in therapeutic contexts [7-9]. The generated iPSCs are extremely similar to embryonic stem cells being capable of generating all cell lineages of different tissues including skin, highlighting a novel method of improving cell-based wound treatment efforts while minimizing ethical concerns and the risk of immunemediated graft rejection $[10,11]$.

We have previously shown that iPSCs-derived keratinocytes (iPSCs-KCs) hold great promise for treating wound healing $[12,13]$. However, the safety profile of these cells is not known and they are potentially limited by the risk of immunogenicity and genetic instability. Recent work has suggested that small membrane-bound exosomes derived from cells can facilitate a substantial fraction of the paracrine signaling necessary to facilitate intracellular communication $[14,15]$. Exosomes of stem cells including bone marrow mesenchymal stem cells, human adipose stem cells, human amniotic epithelial cells and iPSCs can play an anti-inflammatory and anti-fibrosis role, inhibit oxidative stress and enhance angiogenesis, thus promoting skin wound healing [16-20]. As such, we speculate that iPSCs-KCs-derived exosomes (iPSCs-KCs-Eoxs) have the potential to play a direct role in the facilitation of burn wound healing. Up to now, there are no related studies on the iPSCs-KCs-Exos, and there are few studies on the primary keratinocyte's exosomes in skin wound healing [21-23].

Herein, we utilized iPSCs-KCs-Exos to treat murine skin wounds in order to test the therapeutic efficacy of these cell-free vesicles. We found that such iPSCs-KCs-Exos treatment was conducive to more rapid wound closure as well as enhanced endothelial cell and keratinocyte migration. We then evaluated the microRNAs present within these exosomes in an effort to identify important mediators of this wound healing phenotype, and found miR-762 to be highly enriched in iPSCs-KCs-Exos and to serve as an important mediator of cellular migration owing to its ability to target PML. Overall, these data highlight the important roles played by iPSCs-KCs-Exos and miR-762 in the treatment of cutaneous wounds.

\section{Materials And Methods}

\section{Cell culture}

The human iPS cell line was provided by Stem Cell Bank, Chinese Academy of Sciences. hiPSCs were cultured on Matrigel (BD Biosciences, $\mathrm{CA}$ ) in $\mathrm{mTeSR}^{\mathrm{TM}} 1$ medium (Stemcell Technologies, $\mathrm{CA}$ ) and passaged every 4-7 days. hiPSCs were induced to keratinocytes as described previously [11]. Briefly, hiPSCs were switched to unconditioned medium (UM) supplemented with retinoic acid (RA): DMEM/F12 containing $20 \%$ knockout serum replacer, $1 \mathrm{mM}$ L-glutamine, $1 \times$ MEM nonessential amino acids, 0.1 $\mathrm{mM}$ b-mercaptoethanol (all from Life Technologies, USA) and $1 \mathrm{mM}$ all-trans RA (Sigma-Aldrich, USA). Cells were cultured in UM+RA for 7 days, changing medium daily. On day 8, cells were passaged onto gelatin-coated plates in Keratinocyte Defined Serum-free Medium (K-DSFM, Life Technologies). After 4 
weeks, differentiated cells were subcultured using trypsin to obtain high purity keratinocytes populations. Terminal differentiation was induced by adding $1.5 \mathrm{mM} \mathrm{CaCl}_{2}$ in K-DSFM for 10 days. HaCaT cells and HUVECs (COBIOER, China) were cultured in Dulbecco's modified Eagle's medium (DMEM, Gibco, USA), supplemented with $10 \%$ fetal bovine serum (Gibico, USA).

\section{Extraction and identification of iPSCs-KCs-Exos}

iPSCs-KCs were cultured in K-DSFM for $48 \mathrm{~h}$. After collecting the supernatant, exosomes were isolated and purified by serial ultracentrifugation, using ultracentrifuge (Beckman, Germany). The exosomes was suspended in PBS. Concentration of exosomes was measured by Enhanced BCA Protein Assay Kit (Beyotime, China). Then, transmission electron microscopy (TEM) was used to observe the morphological structure of exosomes. Size distribution and concentration of iPSCs-KCs-Exos were analyzed by nanoparticle tracking analysis (NTA) using Nanosight LM 10 (Malvern Panalytical, UK).

\section{Treatment of deep second-degree burns}

The 8-week-old C57BL/6 mice were purchased from the Experimental Animal Center of Southern Medical University. After the hair removal from the back of mice, the opening at one end of a cylindrical plastic tube with a diameter of $1.5 \mathrm{~cm}$ was affixed to the back skin of mice. Then pour boiling water into the tube at a height of $2 \mathrm{~cm}$ and then the tube was removed after 18 seconds. After burn, local subcutaneous injections of PBS or exosomes were given to the wound site.

\section{Histological observations}

The skin tissues were fixed with $4 \%$ paraformaldehyde for $72 \mathrm{~h}$, embedded in paraffin, and then cut into 5 $\mathrm{mm}$ thick sections. The sections were stained with hematoxylin eosin (H\&E) staining (Leagene, China) and Masson's tri-chrome staining (MXB Biotechnologies, China). The re-epithelialization was calculated in the accordance with the formula [distance of the re-epithelium]/ [distance of the wound] $\times 100$.

\section{Immunofluorescence assay}

At room temperature, cells were fixed with $4 \%$ paraformaldehyde for 30 min and then drilled with $0.5 \%$ Triton X-100 for 15 minutes. The cells were incubated overnight at $4^{\circ} \mathrm{C}$ with primary antibodies against K14, loricrin or involucrin (Proteintech, USA) and then incubated with Alexa Fluor 647-conjugated sheep anti-rabbit second antibody (Abcam, UK) for $1 \mathrm{~h}$ at room temperature. DAPI ( $500 \mathrm{ng} / \mathrm{mL}$ ) was used to stain nuclei. Fluorescence was observed under an inverted fluorescence microscope (Leica, Germany)

\section{EDU staining}

After cells were treated for $48 \mathrm{~h}$, EdU was added to the culture medium and incubated for $4 \mathrm{~h}$. The cells were fixed with $4 \%$ paraformaldehyde and then drilled with $0.5 \%$ Triton X-100 for 15 minutes. Then, the cells were stained using Beyoclick ${ }^{\mathrm{TM}}$ EDU cell proliferation kit Alexa Fluor 488 (Beyotime, China) and imaged using an inverted fluorescence microscope (Leica, Germany). 


\section{Wound healing assay}

Cells were seeded into 24-well plates and cultured overnight. After each well was filled with $95 \%$ monolayer of cells, the tip of the $200 \mu \mathrm{L}$ pipette was used to form linear scratch wounds. At $0 \mathrm{~h}$ and $24 \mathrm{~h}$, the wound healing was recorded by a microscope (Leica, Germany). Image J software was used to quantitatively evaluate the wound healing rate.

\section{Transwell assay}

$200 \mu \mathrm{L}$ cell suspension (serum-free DMEM) was added to the upper chamber of Transwell, and $600 \mu \mathrm{L}$ DMEM containing $2 \% \mathrm{FBS}$ was added to the lower chamber. After $24 \mathrm{~h}$ incubation at $5 \% \mathrm{CO}_{2}$ and $37^{\circ} \mathrm{C}$, the cells were fixed with $4 \%$ paraformaldehyde. The cells at the lower side of the membrane were stained with Crystal Violet Staining Solution (Beyotime China) for $15 \mathrm{~min}$. An inverted microscope (Leica, Germany) randomly collected 10 images from each sample.

\section{miRNA microarray analysis}

miRNA microarray analysis was performed by Epibiotek (Guangzhou, China) as previously described [19].

\section{Western blot analysis}

The Western blot assay was performed as previously described [19]. Antibodies used were described as follow: anti-PML, anti-ITGB1 or anti b-actin (all from Abcam, UK).

\section{Reverse transcription quantitative polymerase chain (qRT-PCR)}

Trizo IM $^{\text {TM }}$ reagent (Invitrogen, USA) was used to extract the total RNA from cells, according to the manufacturer's instructions. RNA was reverse-transcribed using RevertAid First Strand cDNA Synthesis Kit (Thermo Scientific, USA). qRT-PCR analysis was using ACEQ Universal SYBR qPCR Master Mix (Vazyme, China). Midtect A TrackTM miRNA qRT-PCR Starter Kit (RiboBio, China) was used for synthesis of miRNA cDNA. Then, Midtect A TrackTM miRNA qRT-PCR Starter Kit (RiboBio, China) were used for qRT-PCR.

\section{Luciferase report assay}

The wild type and mutant miR-762 binding sites of PML 3'UTR sequence were subcloned into psiCHECK2 vector. MiR-762 mimics or negative control mimics were co-transfected with recombinant plasmid into HEK-293 T cells using Lipofectamine 2000 (Invitrogen, USA). According to the manufacturer's instructions, the Firefly and Renilla luciferase activities were detected by Dual Luciferase Reporter Gene Assay Kit (Beyotime, China).

\section{Statistical analysis}

Data were presented as mean \pm standard deviation (SD) of at least three independent experiments ( $n \geq$ 3 ). Statistical analysis was performed by independent samples $t$-test for comparison between two groups 
or one-way ANOVA among the groups. $p<0.05$ was considered statistically significant.

\section{Results}

\section{Characterization of iPSCs-KCs and iPSCs-KCs-Exos.}

To characterize prepared iPSCs-KCs, we conducted immunohistochemical (IHC) staining for cytokeratin14 (K14), which is a keratin marker confirming commitment of the ectoderm to a keratinocyte fate. As shown as in Fig. 1A, K14 was detectable in iPSCs-KCs. In addition, these K14 ${ }^{+}$cells expressed involucrin and loricrin (Fig. 1B), markers of terminal epidermal differentiation, after induced differentiation in high calcium medium $\left(1.5 \mathrm{mM} \mathrm{CaCl}_{2}\right)$, indicating that these iPSCs-KCs have the ability of epidermal differentiation.

Next, iPSCs-KCs-Exos preparations were obtained by ultracentrifuging iPSCs-KCs culture supernatants (Fig. 1C) and analyzing them via transmission electron microscopy (TEM), revealing the presence of exosomes with the expected cup-shaped morphology (Fig. 1D). Nanoparticle tracking analysis (NTA) revealed these particles to have an average diameter of $75 \mathrm{~nm}$ (Fig. 1E). All these data suggested that these nanoparticles were actually exosomes.

\section{Ipscs-kcs-exos Treatment Accelerates The Healing Of Deep Second-degree Burn In Mice}

To test the effects of iPSCs-KCs-Exos treatment on the healing of burn wounds, deep second-degree burns were generated in the abdominal skin of model mice, after which iPSCs-KCs-Exos or an equivalent volume of PBS was locally injected into these areas. Beginning on day 7 post-wounding, significant decreases in wound area in the iPSCs-KCs-Exos group were evident relative to the PBS group, and these improvements grew through day 11 (Fig. 2A). This suggests that iPSCs-KCs-Exos can promote the acceleration of cutaneous wound repair in vivo.

We additionally conducted histological analyses of these healing wounds. Improved re-epithelialization of the wound site was observed in the iPSCs-KCs-Exos treatment group on days 7 and 11 after wounding as compared to the control group when H\&E staining was conducted (Fig. 2B, C). Furthermore, there was a significant increase in the length of epithelial tongues on days 7 and 11 in the iPSCs-KCs-Exos group compared to the PBS group (Fig. 2B, D). Immunohistochemical staining for CD31 further revealed that significant improvements in capillary density were evident in the iPSCs-KCs-Exos-treated wounds.

(Fig. 2E). However, no differences in collagen deposition were observed when comparing iPSCs-KCs-Exos and PBS group (Fig. 2F). Together, these data revealed a role for iPSCs-KCs-Exos as drivers of reepithelialization and angiogenesis, thereby enabling them to accelerate the wound healing process.

iPSCs-KCs-Exos promote the migration of keratinocytes and endothelial cells in vitro 
To better elucidate the mechanisms where by iPSCs-KCs-Exos treatment can accelerate the burn wound healing, the impact of these exosomes on healing-related cell types (endothelial cells and keratinocytes) was next assessed. EdU assays were applied to determine the effect of iPSCs-KCs-Exos on the proliferation of these two kinds of cells. As shown as in Fig. 3A and B, these iPSCs-KCs-Exos did not alter keratinocytes or HUVECs proliferation at all doses in vitro. In a wound healing assay, iPSCs-KCs-Exos treatment was associated with a significantly increased wound closure rate compared to PBS at doses of 1,2 , and $4 \mu \mathrm{g} / \mathrm{ml}$ (Fig. 2C). When evaluated using Transwell assays, HUVECs migratory activity was also found to be significantly increased following iPSCs-KCs-Exos treatment at doses of 1,2, and $4 \mu \mathrm{g} / \mathrm{ml}$ (Fig. 2D). As such, iPSCs-KCs-Exos can enhance the migration of endothelial cells and keratinocytes migration without altering the proliferative activity of these cells.

\section{miR-762 is enriched within iPSCs-KCs-Eoxs and serves as a key mediator of iPSCs-KCs-Exos-induced migration of keratinocytes and endothelial cells}

While microRNAs (miRNAs) account for a relatively small fraction of the total content within extracellular vesicles, they have nonetheless been shown to be important modulators of cellular function [24]. To test the potential relevance of such miRNAs in the context of iPSCs-KCs-Exos-induced migratory activity and wound healing, miRNA microarray analysis was conducted using these exosomes. The result showed that miR-762 was the most abundant miRNA among these detected miRNAs (Table 1). Thus, we focused on exosomal miR-762 for next investigation. In a wound healing assay with $\mathrm{HaCaT}$ cells, the overexpression of miR-762 resulted in enhanced wound closure rates at $24 \mathrm{~h}$ post-wounding (Fig. 4A). To further confirm the functional importance of this miRNA, iPSCs-KCs-Exos-treated HaCaT cells were treated with a specific inhibitor of miR-762, which attenuated this enhanced migratory activity (Fig. 4A). The same effect was also observed in HUVECs (Fig. 4B). In addition, the expression of miR-762 in HaCaT cells and HUVECs was indeed significantly elevated at $48 \mathrm{~h}$ post-iPSCs-KCs-Exos treatment, confirming the ability of these exosomes to deliver miR-762 to recipient cells (Fig. 4C). These data thus suggest that miR-762 is an important mediator of iPSCs-KCs-Exos-induced migration of keratinocyte and endothelial cells in vitro. 
Table 1

Highly expressed miRNAs in iPSCs-KCs-Exos.

\begin{tabular}{|lll|}
\hline Gene ID & Name & Read count \\
\hline MIMAT0010313 & hsa-miR-762 & 115546 \\
\hline MIMAT0000266 & hsa-miR-205-5p & 114148 \\
\hline MIMAT0000067 & hsa-let-7f-5p & 91099 \\
\hline MIMAT0000069 & hsa-miR-16-5p & 51004 \\
\hline MIMAT0000086 & hsa-miR-29a-3p & 28959 \\
\hline MIMAT0000278 & hsa-miR-221-3p & 24733 \\
\hline MIMAT0000415 & hsa-let-7i-5p & 22310 \\
\hline MIMAT0000062 & hsa-let-7a-5p & 14970 \\
\hline MIMAT0000063 & hsa-let-7b-5p & 13934 \\
\hline MIMAT0005898 & hsa-miR-1246 & 13716 \\
\hline MIMAT0000732 & hsa-miR-378a-3p & 13533 \\
\hline MIMAT0000076 & hsa-miR-21-5p & 13026 \\
\hline MIMAT0000259 & hsa-miR-182-5p & 12760 \\
\hline MIMAT0000281 & hsa-miR-224-5p & 11130 \\
\hline MIMAT0000083 & hsa-miR-26b-5p & 10781 \\
\hline MIMAT0000226 & hsa-miR-196a-5p & 9398 \\
\hline MIMAT0000764 & hsa-miR-339-5p & 9122 \\
\hline MIMAT0000078 & hsa-miR-23a-3p & 8538 \\
\hline MIMAT0049032 & hsa-miR-12136 & 8013 \\
\hline MIMAT0000100 & hsa-miR-29b-3p & 6287 \\
\hline
\end{tabular}

\section{Mir-762 Targets Pml To Regulate The Migratory Activity Of Keratinocytes And Endothelial Cells Through Itgb1}

To further clarify the regulatory role of iPSCs-KCs-Exos-derived miR-762 in the context of wound healing, potential target genes for this miRNA were next identified using the Targetscan, miRDB, and microRNA.org databases. This approach identified PML as a putative target of this miRNA. To confirm this prediction, luciferase reporter constructs containing wild-type (WT) or mutated (MUT) versions of the putative miR-762 binding site located within the PML 3'-untranslated region (UTR) were prepared 
(Fig. 5A). When 293T cells were transfected with these reporters and miR-762 mimics, this miRNA was confirmed to suppress the activity of the WT but not the MUT reporter, confirming the predicted regulatory relationship (Fig. 5B). Moreover, mRNA and protein levels of PML were significantly lower in HaCaT cells and HUVECs following miR-762 mimic or iPSCs-KCs-Exos treatment (Fig. 5C, D). PML is thus a direct miR762 target gene. To further examine the link between PML downregulation and the effects of miR-762 and iPSCs-KCs-Exos on the migration of keratinocytes and endothelial cells, the PML was overexpressed with pcDNA3.1 vector. As shown as in Fig. 5E, overexpression of PML inhibited the migration of HaCaT cells and could restraine the cell migration that was promoted by miR-762 mimics or iPSCs-KCs-Exos. The similar results were also observed in HUVECs (Fig. 5F). Taken together, above results suggest that miR-762 and iPSCs-KCs-Exos induced acceleration of keratinocytes and endothelial cells is mediated at least partly by inhibiting PML expression.

\section{PML regulated ITGB1 is involved in the promotion of keratinocytes and endothelial cells migration by miR-762 and iPSCs-KCs-Exos.}

Reineke et al. previously demonstrated that ITGB1 was responsible for mediating PML-induced changes in migratory activity [25]. In this study, we examined the effects of PML knockdown on ITGB1 expression by siRNA. When PML expression decreased, we observed an increase in ITGB1 mRNA and protein expression in keratinocytes and endothelial cells (Fig. 6A, B). We further found that the expression of ITGB1 also increased after treatment with miR-762 mimic or iPSCs-KCs-Eoxs (Fig. 6C, D). Moreover, siRNA-mediated ITGB1 knockdown ablated the miR-762 mimic and iPSCs-KCs-Eoxs induced migration of HaCaT cells and HUVECs (Fig. 6E, F). Together, these data suggest that miR-762 can target PML to enhance the migration of keratinocyte and endothelial cells through a mechanism involving ITGB1.

\section{Discussion}

Herein, we found that iPSCs-KCs-Exos treatment was sufficient to promote keratinocyte migration and to thereby accelerate the healing of deep second-degree burn wounds in mice. We additionally determined that the most abundant miRNA within these exosomes, miR-762, was a key mediator of this enhanced migratory effect owing to its ability to target PML.

In the process of wound healing, keratinocytes, fibroblasts and endothelial cells are the main cell populations involved in re-epithelialization and granulation tissue construction [26]. Therefore, communication between these cells plays a key role in skin repair. This network is likely regulated by cellderived exosomes, which carry a range of macromolecular cargos that can be delivered to target cells, enabling them to regulate recipient cell responses in a variety of complex manners $[14,15]$. Both HaCaT cells and primary keratinocytes can release exosomes and other extracellular vesicles [27, 28]. In their deep sequencing analysis of microRNAs within keratinocyte-derived extracellular vesicles, Than et al. found that the most enriched miRNAs found therein were linked to key physiological processes such as fibroblast migration and dermal-epithermal wound healing, suggesting a role for these vesicles in the context of cutaneous wound healing [21]. 
This study was designed to explore the ability of iPSC-KC-derived exosomes to regulate the process of burn wound healing. We successfully generated and characterized iPSCs-KCs-Exos, which were of the expected size and exhibited characteristic morphological features. Cutaneous wound healing processes are complicated and necessitate interplay between inflammatory, angiogenic, remodeling, proliferative, epithelialization, and fibrotic processes [29]. Histological analyses revealed that increases in reepithelialization and capillary density were evident in wound sites following iPSCs-KCs-Exos treatment, but collagen formation was not affected. Thus, we then further examined the effects of these exosomes on endothelial cells and keratinocytes, which are the key mediators of angiogenesis and epithelialization, respectively. These analyses revealed that iPSCs-KCs-Exos significantly enhanced the migratory activity of these cells without impacting their proliferative activity. As such, the beneficial impact of these exosomes in the context of cutaneous wound healing may be primarily attributable to their ability to promote keratinocyte and endothelial cell migration.

Exosomes contain a variety of miRNAs, mRNAs, lipids, and proteins that are compiled within the ExoCarta database $[14,15]$. Of these, miRNAs are the best-studied exosomal cargos owing to their ability to regulate target mRNA expression. Herein, we detected many miRNAs that were highly abundant within iPSCs-KCs-Exos, of which miR-762 was detected at the highest levels. Moreover, we found that miR-762 was able to promote endothelial cell and keratinocyte migration in a manner similar to iPSCs-KCs-Exos treatment, suggesting that it is likely a primary mediator of the phenotypic effects-KCs-Exos in the context of wound healing. In prior research, miR-762 has been found to regulate a range of target genes in different contexts. The upregulation of miR-762 in non-small cell lung cancer, bladder cancer, breast cancer and nasopharyngeal carcinoma has been linked to enhanced tumor cell invasivity, migration, and proliferation [30-33]. However, there have been no prior studies regarding the role of this specific miRNA in keratinocytes or endothelial cells. In line with these past findings, we observed a pro-migratory role for miR-762 in both of these cell types.

Herein, we identified promyelocytic leukemia (PML) as a novel miR-762 target gene associated with the ability of this miRNA to regulate endothelial cell and keratinocyte migration. PML plays a role in a number of important cellular processes, including tumor suppression, apoptosis, transcriptional regulation, DNA damage response, senescence and viral defense mechanisms [34-36]. Herein, we confirmed that PML was a direct miR-762 target, and we found that overexpressing PML was sufficient to reverse the beneficial impact of miR-762 on cellular migration. In prior reports, the ability of PML to suppress the migration of MDA-MB-231 cells was attributed to its ability to promote ITGB1 downregulation [25]. ITGB1 are important for attachment to and integrity of cell-extracellular matrix (BME) interaction by mediating binding to BME components, and they are generally required for migration of keratinocytes and endothelial cells $[37,38]$. We thus explored the link between ITGB1 and this miR-762/PML regulatory axis, leading us to conclude that PML overexpression suppressed ITGB1 expression while miR-762 was able to reverse this effect. We further confirmed a role for ITGB1 in the miR-762/PML-mediated migratory phenotypes observed in this experimental system. However, as miRNAs target multiple genes, these results do not offer any insight into the ability of miR-762 to regulate other signaling pathways within 
keratinocytes. Moreover, while PML and ITGB1 clearly play a role in this process, further work is required to elucidate the underlying mechanisms.

In conclusion, these results indicate that iPSCs-KCs-Exos treatments can significantly improve burn wound healing outcomes, highlighting novel approaches to expediting cutaneous wound healing and providing a foundation for future research efforts.

\section{Abbreviations}

iPSCs

induced pluripotent stem cells

iPSCs-KCs

iPSCs-derived keratinocytes

iPSCs-KCs-Exos

iPSCs-KCs-derived exosomes

PML

promyelocytic leukemia

integrin beta1 (ITGB1)

UM

unconditioned medium

RA

retinoic acid

K-DSFM

keratinocyte defined serum-free Medium

DMEM

Dulbecco's modified eagle's medium

HUVECS

human umbilical vein endothelial cells

PBS

phosphate-buffered saline

TEM

transmission electron microscopy

NTA

nanoparticle tracking analysis

$H \& E$

hematoxylin and eosin

DAPI

4,6-diamidino-2-phenylindole

EdU

5-Ethynyl-2'-deoxyuridine

qRT-PCR 
quantitative real-time polymerase chain reaction

K14

cytokeratin-14

miRNAs

microRNAs

BME

cell-extracellular matrix.

\section{Declarations}

\section{Acknowledgements}

Not applicable.

\section{Authors' Contributions}

L.Z. and Y.Y. participated in all aspects of the study, including design, financial support, analyses, final approval of manuscript and revised the manuscript. YY. B. and LJ. Y. performed the experimental work and analyzed the data. BT. L., GP. T. and CX. L. performed the collection and/or assembly of data and revised the manuscript; All authors agreed to be accountable for all aspects of the work.

\section{Funding}

This work was funded by the National Natural Science Foundation of China (No: 82072186, No: 81872514).

\section{Availability of data and materials}

The authors agree to share data and materials related to this manuscript.

\section{Ethics approval and consent to participate}

The Bioethics Committee of Southern Medical University approved all animal procedures, which were in accordance with the National Institutes of Health $(\mathrm{NIH})$ Guide for the Care and Use of Laboratory Animals.

\section{Consent for publication}

Not applicable.

\section{Competing Interests}

The authors declared no potential conflicts of interest. 


\section{References}

1. Jeschke MG, van Baar ME, Choudhry MA, Chung KK, Gibran NS, Logsetty S. Burn injury. Nat Rev Dis Primers 2020 Feb 13;6(1):11.

2. Nielson CB, Duethman NC, Howard JM, Moncure M, Wood JG. Burns: Pathophysiology of Systemic Complications and Current Management. J Burn Care Res. 2017 Jan/Feb;38(1):e469-e481.

3. Roshangar L, Soleimani Rad J, Kheirjou R, Reza Ranjkesh M, Ferdowsi Khosroshahi A. Skin Burns: Review of Molecular Mechanisms and Therapeutic Approaches. Wounds. 2019 Dec;31(12):308-315.

4. Ter Horst B, Chouhan G, Moiemen NS, Grover LM. Advances in keratinocyte delivery in burn wound care. Adv Drug Deliv Rev. 2018 Jan 1;123:18-32.

5. Rowan MP, Cancio LC, Elster EA, Burmeister DM, Rose LF, Natesan S, Chan RK, Christy RJ, Chung KK. Burn wound healing and treatment: review and advancements. Crit Care. 2015 Jun 12;19:243.

6. Fang T, Lineaweaver WC, Sailes FC, Kisner C, Zhang F. Clinical application of cultured epithelial autografts on acellular dermal matrices in the treatment of extended burn injuries. Ann Plast Surg. 2014 Nov;73(5):509-515.

7. Takahashi K, Yamanaka S. Induction of pluripotent stem cells from mouse embryonic and adult fibroblast cultures by defined factors. Cell. 2006 Aug 25;126(4):663-676.

8. Talug B, Tokcaer-Keskin Z. Induced Pluripotent Stem Cells in Disease Modelling and Regeneration. Adv Exp Med Biol. 2019;1144:91-99.

9. Hu J, Wang J. From embryonic stem cells to induced pluripotent stem cells-Ready for clinical therapy? Clin Transplant. 2019 Jun;33(6):e13573.

10. Miyake T, Shimada M. 3D Organoid Culture Using Skin Keratinocytes Derived from Human Induced Pluripotent Stem Cells. Methods Mol Biol. 2021 Mar 11.

11. Selekman JA, Grundl NJ, Kolz JM, Palecek SP. Efficient generation of functional epithelial and epidermal cells from human pluripotent stem cells under defined conditions. Tissue Eng Part C Methods. 2013 Dec;19(12):949-960.

12. Yan $Y$, Jiang J, Zhang $M$, Chen $Y$, Wang $X$, Huang $M$, Zhang L. Effect of iPSCs-derived keratinocytes on healing of full-thickness skin wounds in mice. Exp Cell Res. 2019 Dec 1;385(1):111627.

13. Wu R, Du D, Bo Y, Zhang M, Zhang L, Yan Y. Hsp90alpha promotes the migration of iPSCs-derived keratinocyte to accelerate deep second-degree burn wound healing in mice. Biochem Biophys Res Commun. 2019 Nov 26;520(1):145-151.

14. Kalluri R, LeBleu VS. The biology, function, and biomedical applications of exosomes. Science. 2020 Feb 7;367(6478):eaau6977.

15. Pegtel DM, Gould SJ. Exosomes. Annu Rev Biochem. 2019 Jun 20;88:487-514.

16. He L, Zhu C, Jia J, Hao XY, Yu XY, Liu XY, Shu MG. ADSC-Exos containing MALAT1 promotes wound healing by targeting miR-124 through activating Wnt/ $\beta$-catenin pathway. Biosci Rep. 2020 May 29;40(5):BSR20192549. 
17. Ren S, Chen J, Duscher D, Liu Y, Guo G, Kang Y, Xiong H, Zhan P, Wang Y, Wang C, Machens HG, Chen Z. Microvesicles from human adipose stem cells promote wound healing by optimizing cellular functions via AKT and ERK signaling pathways. Stem Cell Res Ther. 2019 Jan 31;10(1):47.

18. An Y, Lin S, Tan X, Zhu S, Nie F, Zhen Y, Gu L, Zhang C, Wang B, Wei W, Li D, Wu J. Exosomes from adipose-derived stem cells and application to skin wound healing. Cell Prolif. 2021 Mar;54(3):e12993.

19. Yan Y, Wu R, Bo Y, Zhang M, Chen Y, Wang X, Huang M, Liu B, Zhang L. Induced pluripotent stem cells-derived microvesicles accelerate deep second-degree burn wound healing in mice through miR16-5p-mediated promotion of keratinocytes migration. Theranostics. 2020 Aug 8;10(22):9970-9983.

20. Rani S, Ritter T. The Exosome - A Naturally Secreted Nanoparticle and its Application to Wound Healing. Adv Mater. 2016 Jul;28(27):5542-5552.

21. Than UTT, Guanzon D, Broadbent JA, Leavesley DI, Salomon C, Parker TJ. Differential Expression of Keratinocyte-Derived Extracellular Vesicle Mirnas Discriminate Exosomes From Apoptotic Bodies and Microvesicles. Front Endocrinol (Lausanne). 2018 Sep 11;9:535.

22. Zhou X, Brown BA, Siegel AP, El Masry MS, Zeng X, Song W, Das A, Khandelwal P, Clark A, Singh K, Guda PR, Gorain M, Timsina L, Xuan Y, Jacobson SC, Novotny MV, Roy S, Agarwal M, Lee RJ, Sen CK, Clemmer DE, Ghatak S. Exosome-Mediated Crosstalk between Keratinocytes and Macrophages in Cutaneous Wound Healing. ACS Nano. 2020 Oct 27;14(10):12732-12748.

23. Chavez-Muñoz C, Kilani RT, Ghahary A. Profile of exosomes related proteins released by differentiated and undifferentiated human keratinocytes. J Cell Physiol. 2009 Oct;221(1):221-231.

24. Yu X, Odenthal M, Fries JW. Exosomes as miRNA Carriers: Formation-Function-Future. Int J Mol Sci. 2016 Dec 2;17(12):2028.

25. Reineke EL, Liu Y, Kao HY. Promyelocytic leukemia protein controls cell migration in response to hydrogen peroxide and insulin-like growth factor-1. J Biol Chem. 2010 Mar 26;285(13):9485-9492.

26. Rodrigues M, Kosaric N, Bonham CA, Gurtner GC. Wound Healing: A Cellular Perspective. Physiol Rev. 2019 Jan 1;99(1):665-706.

27. Ogawa M, Udono M, Teruya K, Uehara N, Katakura Y. Exosomes Derived from Fisetin-Treated Keratinocytes Mediate Hair Growth Promotion. Nutrients. 2021 Jun 18;13(6):2087.

28. Jiang M, Fang H, Shao S, Dang E, Zhang J, Qiao P, Yang A, Wang G. Keratinocyte exosomes activate neutrophils and enhance skin inflammation in psoriasis. FASEB J. 2019 Dec;33(12):13241-13253.

29. Sorg H, Tilkorn DJ, Hager S, Hauser J, Mirastschijski U. Skin Wound Healing: An Update on the Current Knowledge and Concepts. Eur Surg Res. 2017;58(1-2):81-94.

30. Ge P, Cao L, Chen X, Jing R, Yue W. miR-762 activation confers acquired resistance to gefitinib in nonsmall cell lung cancer. BMC Cancer. 2019 Dec 10;19(1):1203.

31. Liu T, Lu Q, Liu J, Xie S, Feng B, Zhu W, Liu M, Liu Y, Zhou X, Sun W, Zhang Y, Chen X, Fang F, Guo H, Yang R. Circular RNA FAM114A2 suppresses progression of bladder cancer via regulating NP63 by sponging miR-762. Cell Death Dis. 2020 Jan 22;11(1):47. 
32. Li Y, Huang R, Wang L, Hao J, Zhang Q, Ling R, Yun J. microRNA-762 promotes breast cancer cell proliferation and invasion by targeting IRF7 expression. Cell Prolif. 2015 Dec;48(6):643-649.

33. Bao LH, Ji K, Li D, Liu SS, Song ZY, Xia GH. The biological function and diagnostic value of miR-762 in nasopharyngeal carcinoma. J Chin Med Assoc. 2021 May 1;84(5):498-503.

34. Guan D, Kao HY. The function, regulation and therapeutic implications of the tumor suppressor protein, PML. Cell Biosci. 2015 Nov 4;5:60.

35. Jin G, Gao Y, Lin HK. Cytoplasmic PML: from molecular regulation to biological functions. J Cell Biochem. 2014 May;115(5):812-818.

36. Imani-Saber Z, Ghafouri-Fard S. Promyelocytic leukemia gene functions and roles in tumorigenesis. Asian Pac J Cancer Prev. 2014;15(19):8021-8028.

37. Koivisto L, Heino J, Häkkinen L, Larjava H. Integrins in Wound Healing. Adv Wound Care (New Rochelle). 2014 Dec 1;3(12):762-783.

38. Brakebusch C, Fässler R. beta 1 integrin function in vivo: adhesion, migration and more. Cancer Metastasis Rev. 2005 Sep;24(3):403-411.

\section{Figures}


A
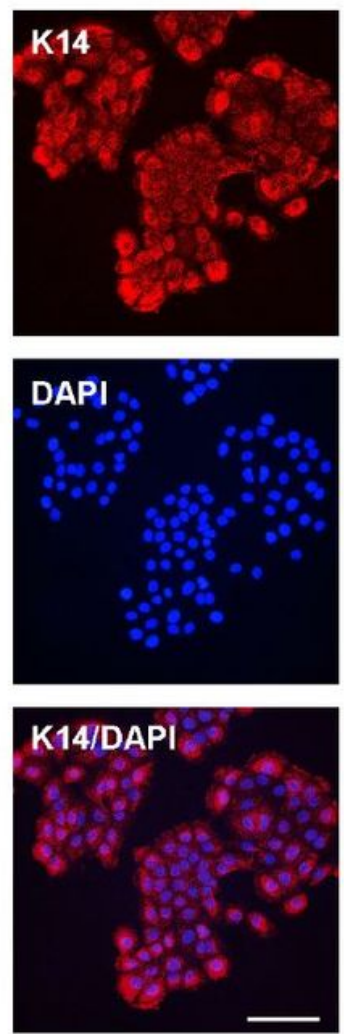

B
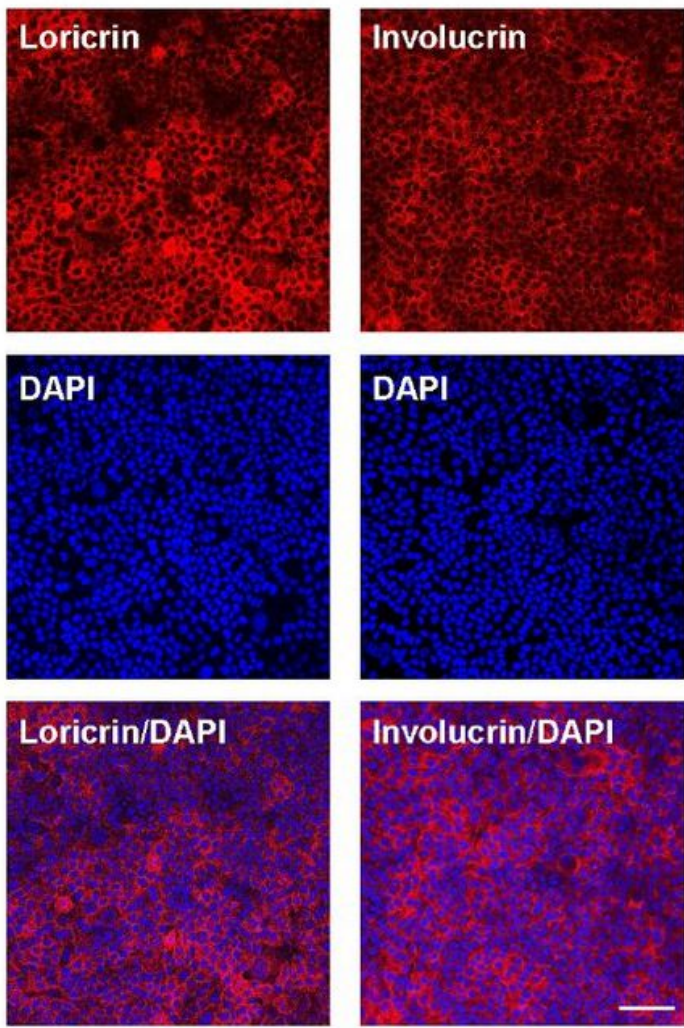
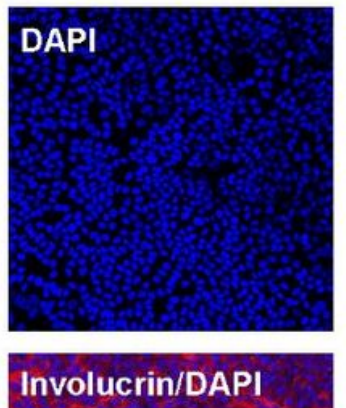

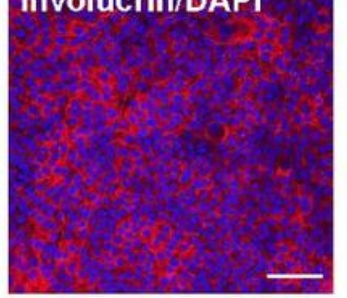

$\mathbf{E}$

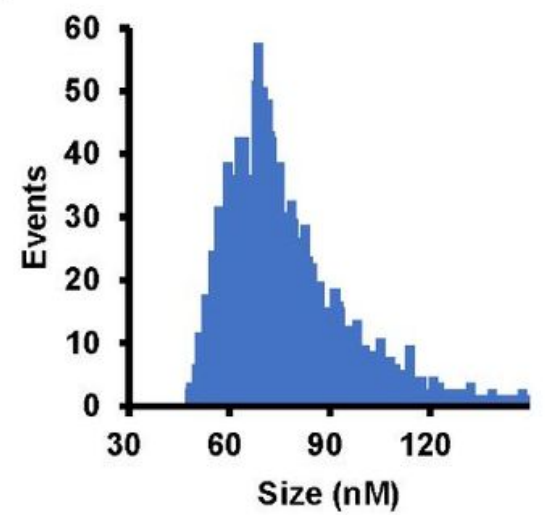

\section{C}

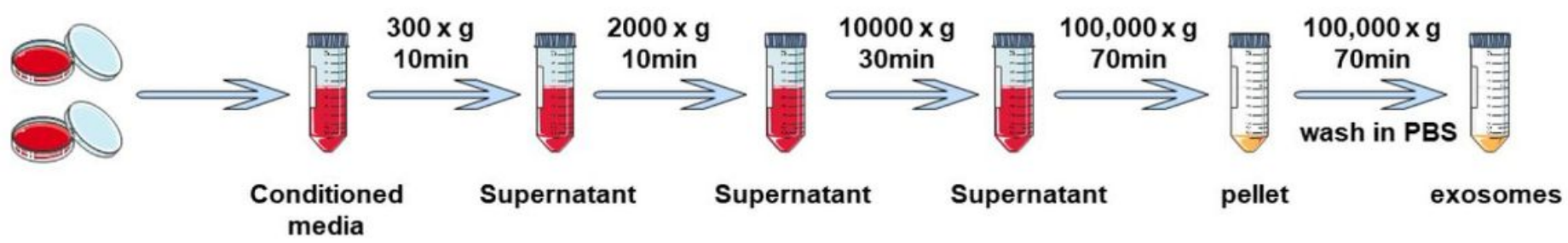

Figure 1

Characterization of iPSCs-KCs and iPSCs-KCs-Exos. A Representative images of immunohistochemical staining for K14 in iPSCs-KCs. Scale bar, $100 \mu \mathrm{m}$. B Representative images of immunohistochemical staining for involucrin and loricrin in iPSCs-KCs culture for 10 days in DKSFM supplemented with 1.5 $\mathrm{mmol} / \mathrm{L}$ of $\mathrm{CaCl}_{2}$. Scale bar, $100 \mu \mathrm{m}$. Nuclei was stained by DAPI (blue). C Schematic diagram of iPSCsKCs-Exos extraction process. D The transmission electron microscopy (TEM) image of iPSCs-KCs-Exos. Scale bar, $100 \mathrm{~nm}$. E Nanoparticle tracking analysis (NTA) result of iPSCs-KCs-Exos. 
A
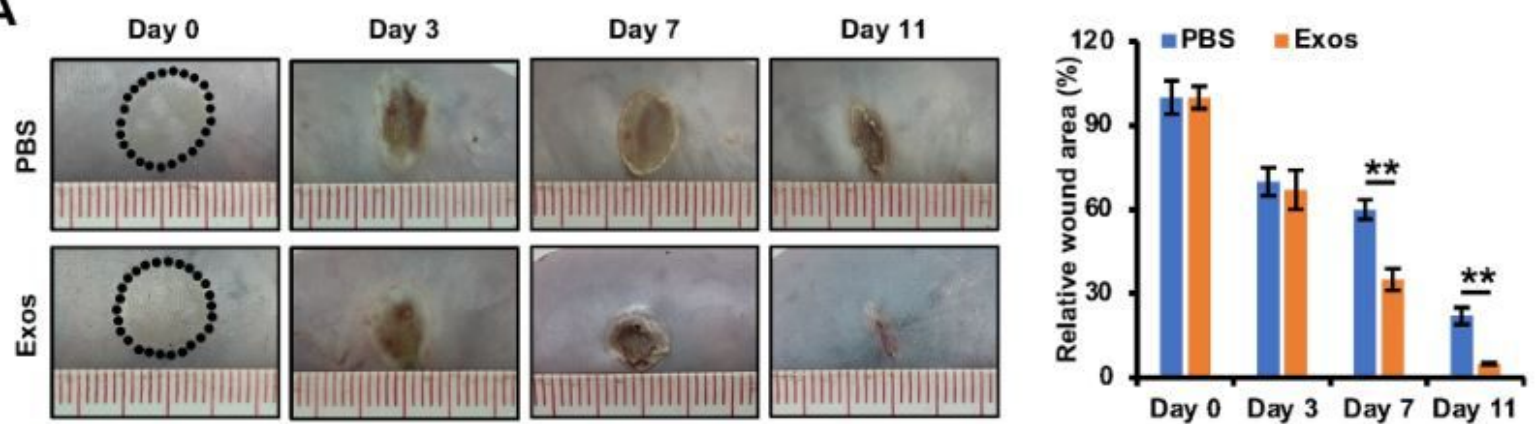

B

PBS

Exos
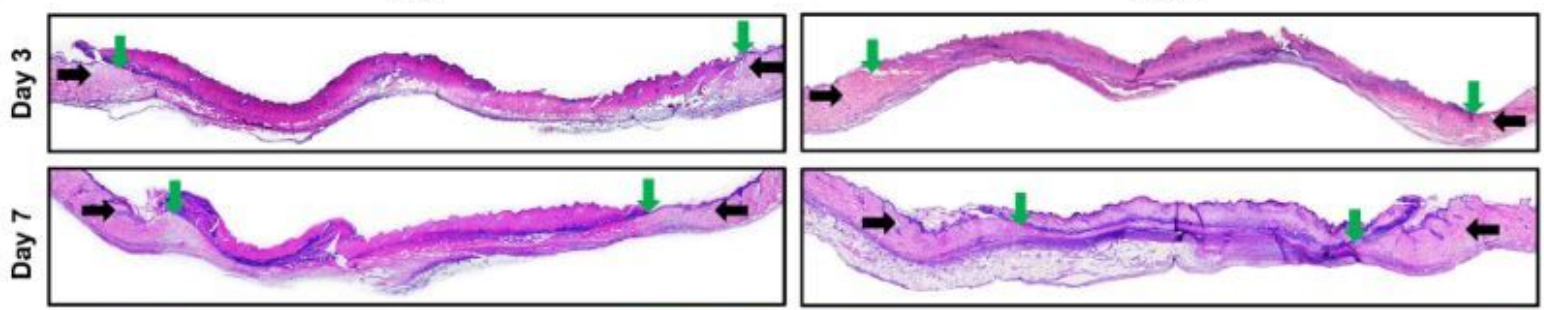

risin

L

$\sqrt{2.3 .3}$

Figure 2

iPSCs-KCs-Exos accelerate deep second-degree burn wound healing in vivo. A Representative images of wounds treated with PBS or iPSCs-KCs-Exos on days $0,3,7$ and 11 after burning and quantitative analysis of wound area in each group. B H\&E staining of wounded skin sections treated with PBS or iPSCs-KCs-Exos on days 3, 7 and 11 after burning. Black arrows indicate the dermal border; green arrows indicate the epidermal margin. Scale bar, $400 \mu \mathrm{m}$. C Quantitative analysis of the re-epithelialization ration 
of wounds in each group. D Quantitative analysis of the length of epithelial tongues of wounds in each group. E Immunohistochemistry staining for CD31 wounds and quantitative analysis of the numbers of stained capillaries in each group on days 11 after burning. Scale bar, $50 \mu \mathrm{m}$. F Masson's trichrome staining of wounds in each group on days 7 and 11 after burning. Scale bar, $50 \mu \mathrm{m} .{ }^{\star} P<0.05, * \star P<0.01$.

A

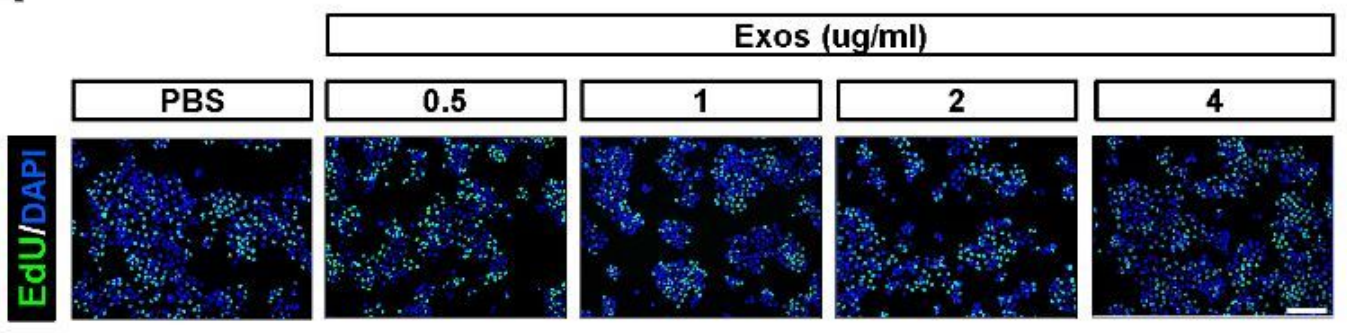

B

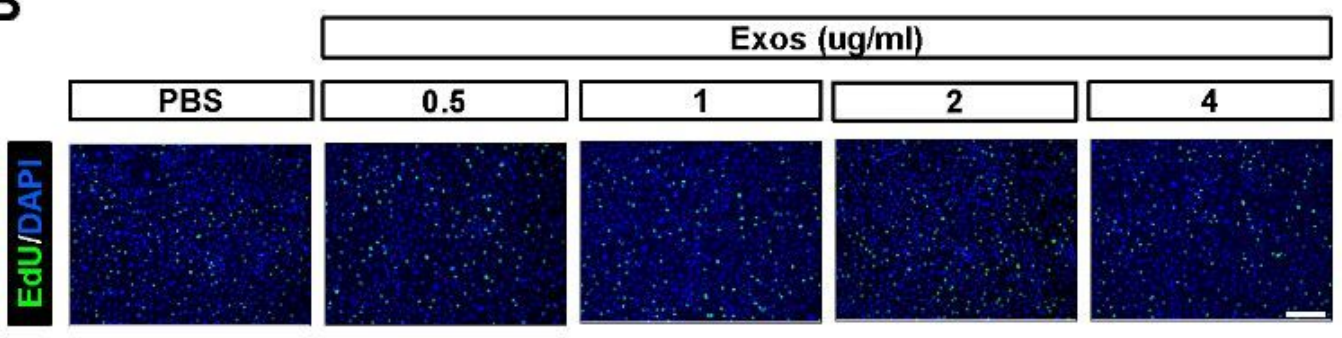

C

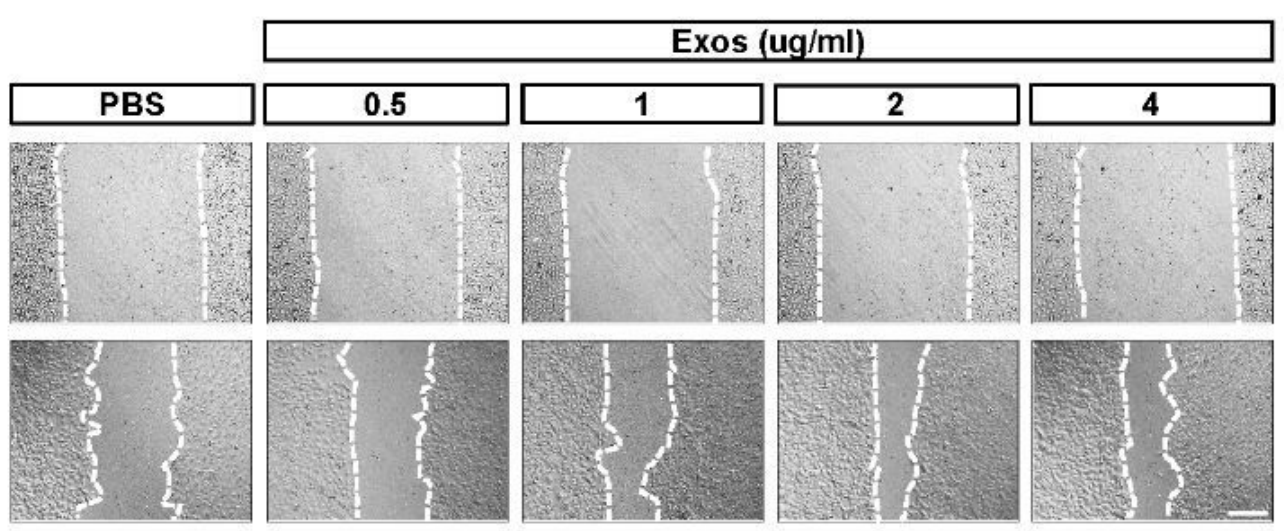

D

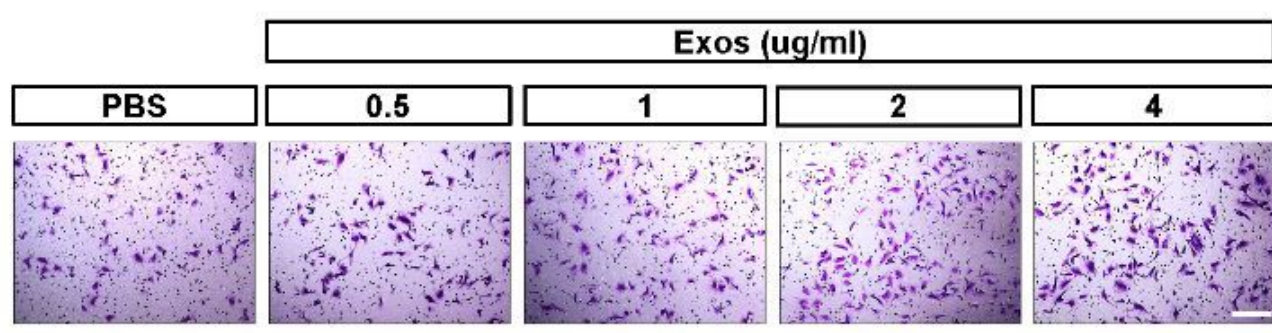

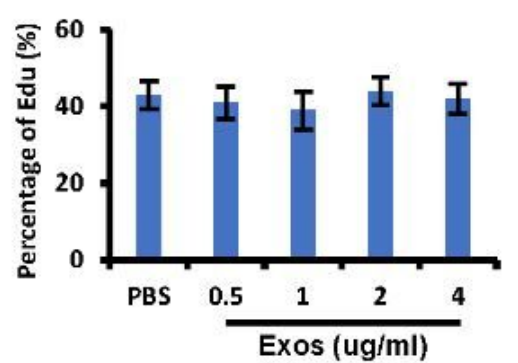
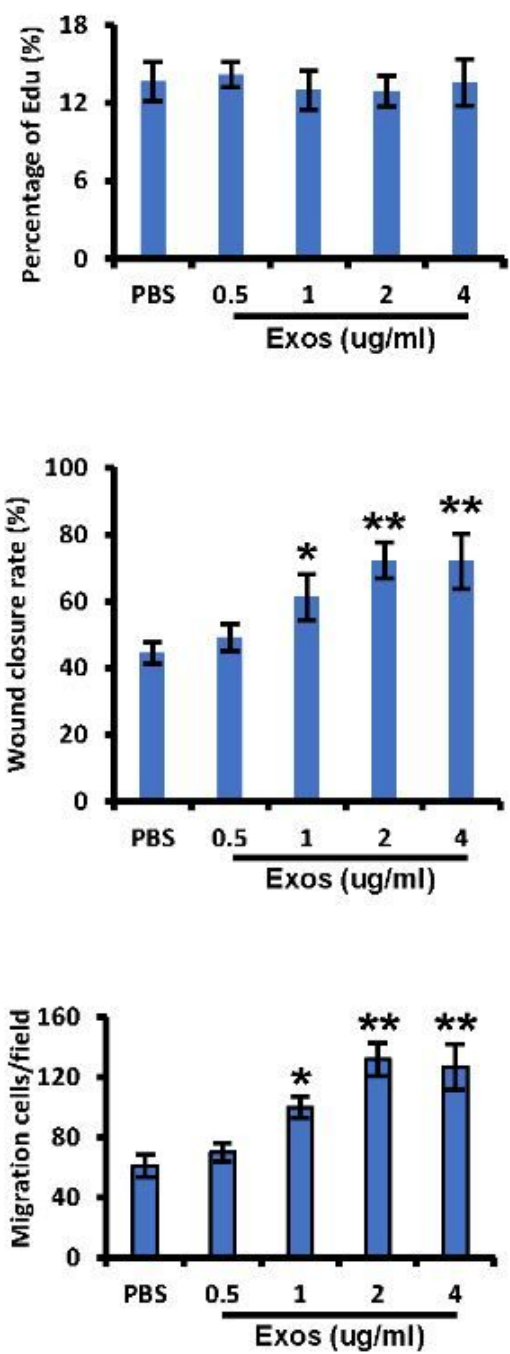

Figure 3 
iPSCs-KCs-Exos promote the migration of keratinocytes and endothelial cells in vitro. A Representative fluorescence images of EdU staining of HaCaT cells treated with PBS or iPSCs-KCs-Exos $(0.5,1,2$, or 4 $\mu \mathrm{g} / \mathrm{mL}$ ) and quantitative analysis of the proliferation rates in each group. Scale bar, $200 \mu \mathrm{m}$. B Representative fluorescence images of EdU staining of HUVECs and quantitative analysis of the proliferation rates in each group. Scale bar, $200 \mu \mathrm{m}$. C Representative images of the wound healing assay and quantitative analysis of the wound healing rates in each group at $24 \mathrm{~h}$. Scale bar, $200 \mu \mathrm{m}$. D Images of migrated HUVECs and quantitative analysis of the migrated cells in each group. Scale bar, $200 \mu \mathrm{m} . * P$ $<0.05,{ }^{\star} * P<0.01$.

A
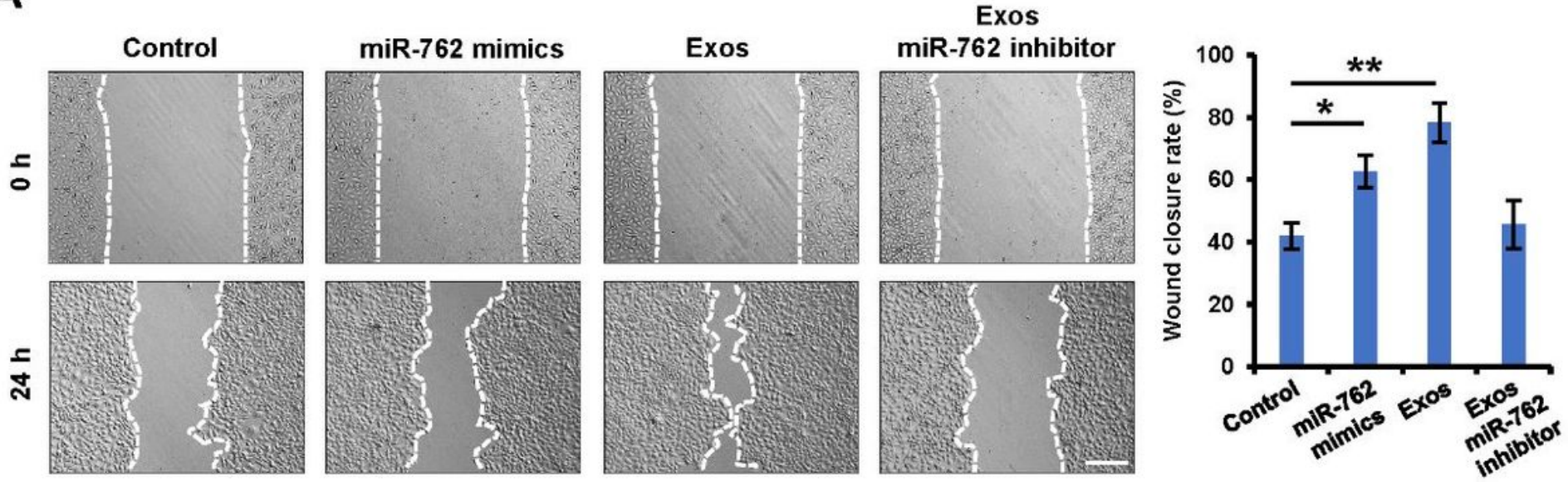

B
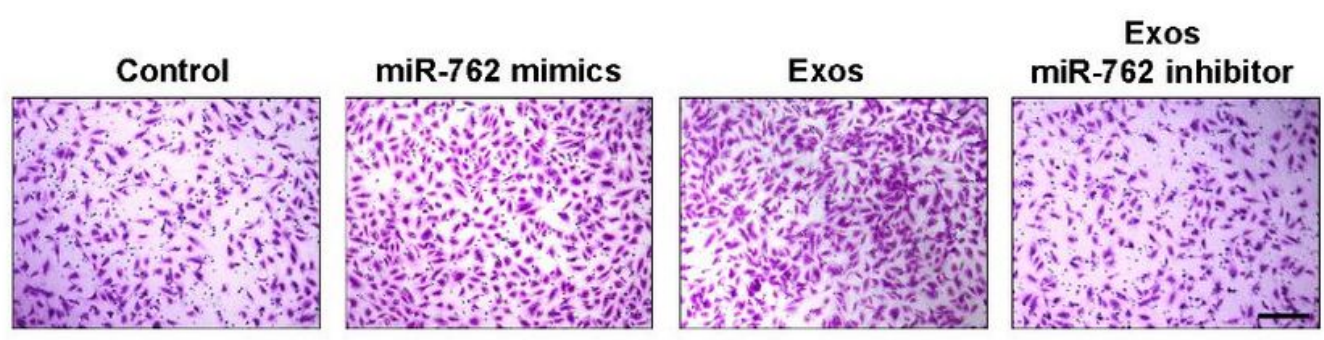

C
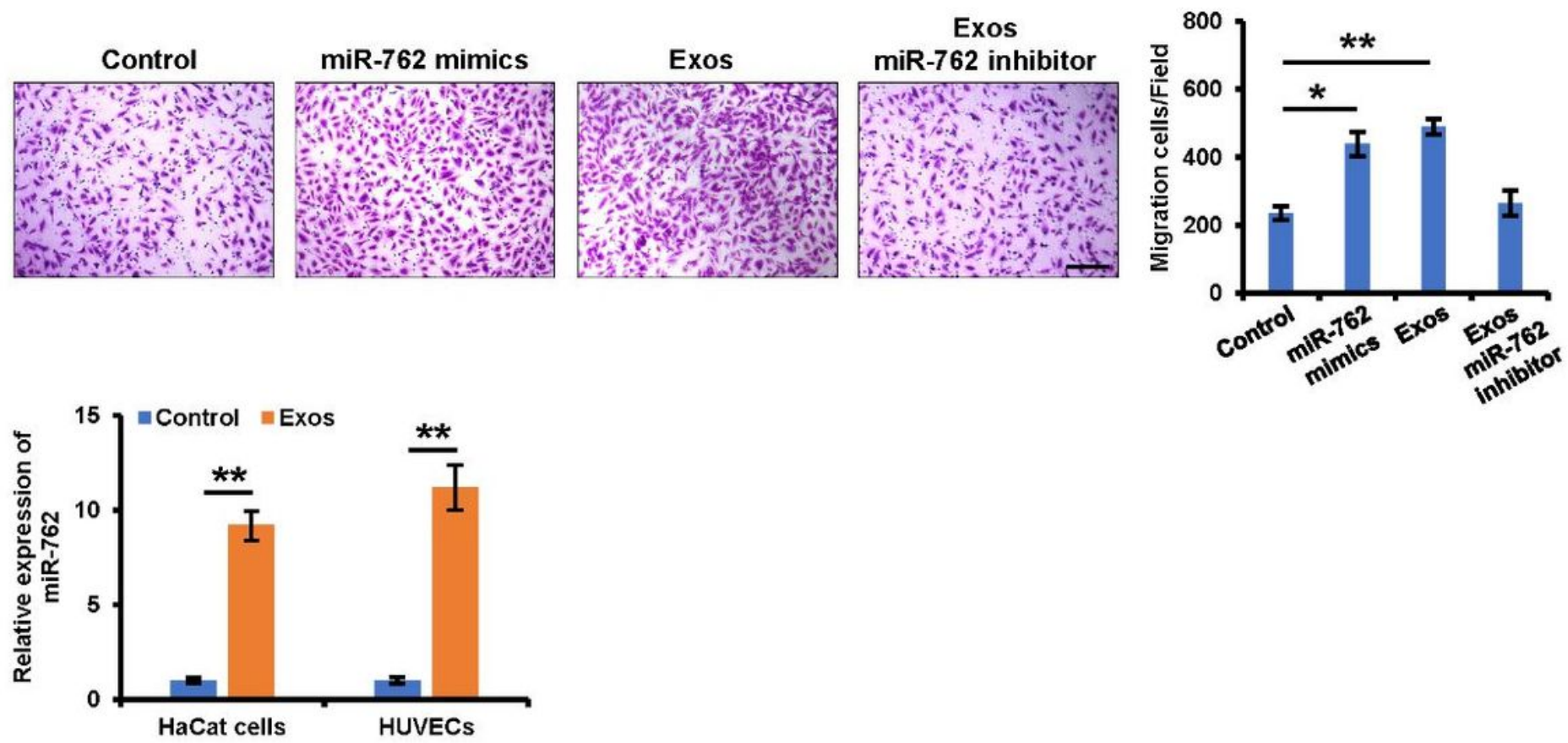

Figure 4 
Mir-762 is a key mediator of iPSCs-KCs-Exos induced migration of keratinocytes and endothelial cells. A Representative images of the wound healing assay of keratinocytes in each group (left panel). Scale bar $=100 \mu \mathrm{m}$. Quantitative analysis of the wound healing rates in each group (right panel). B Images of migrated HUVECs in each group. Scale bar, $200 \mu \mathrm{m}$. Quantitative analysis of the migrated cells (right panel). Scale bar, $200 \mu \mathrm{m}$. C qRT-PCR analysis of miR-762 expression in HaCaT cells and HUVECs which were treated with iPSCs-KCs-Exos $(2 \mu \mathrm{g} / \mathrm{mL})$ for $24 \mathrm{~h} .{ }^{*} P<0.05$, $* * P<0.01$.

A

miR-762 3'-CGAGCCGGGGCCGGGGUCGGGG-5' WT PML 3'UTR 5'-CCCAGGUCCUGGUACCCAGCCCU-3' MUT PML 3'UTR 5'-CCCAGGUCCUGGUACAACUAUAU-3'
B

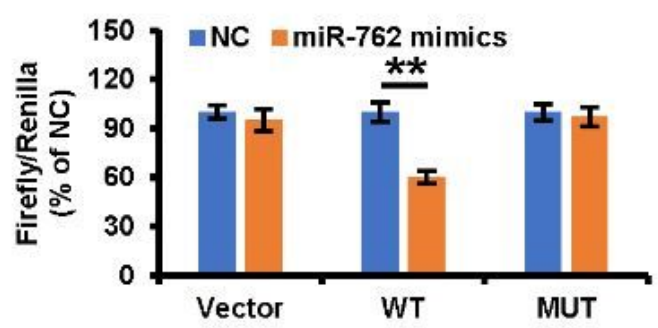

C

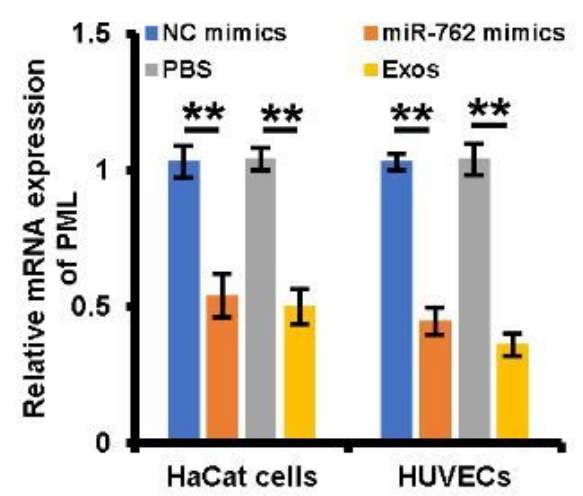

E

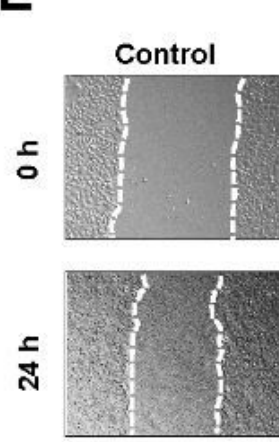

miR-762 mimics

F
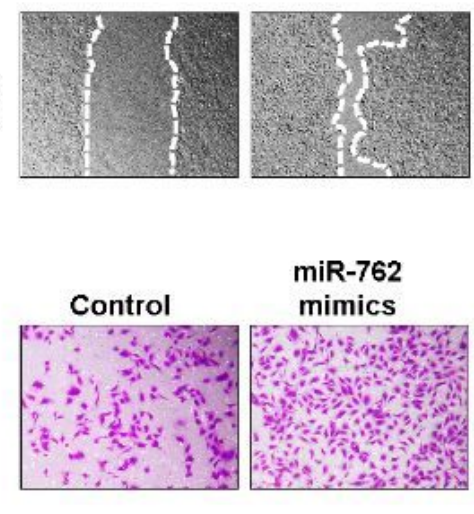

miR-762

mimics
D
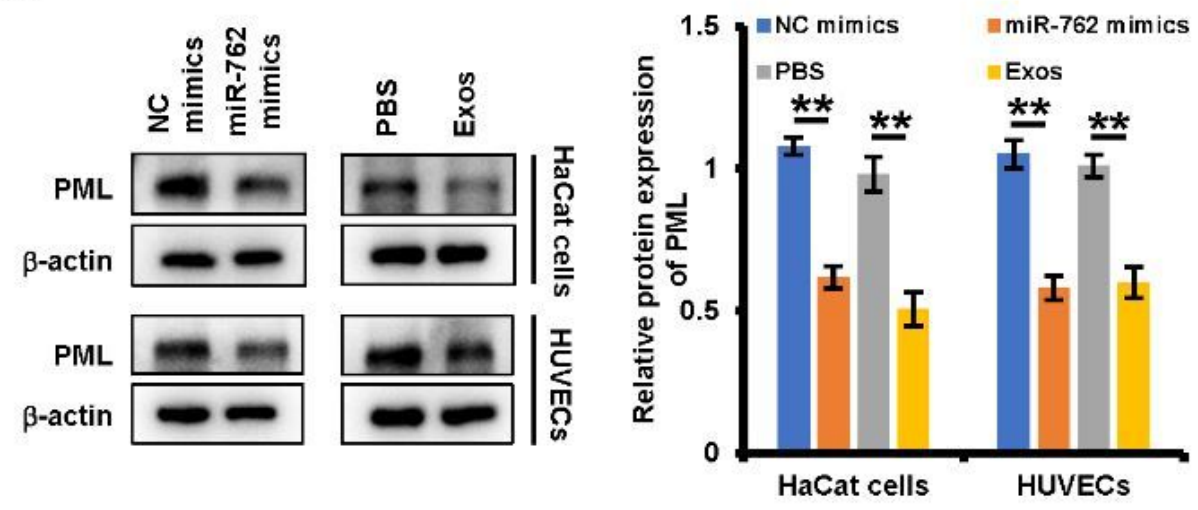
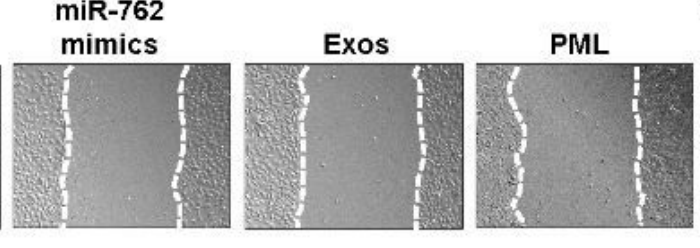

miR-762 mimics + PML
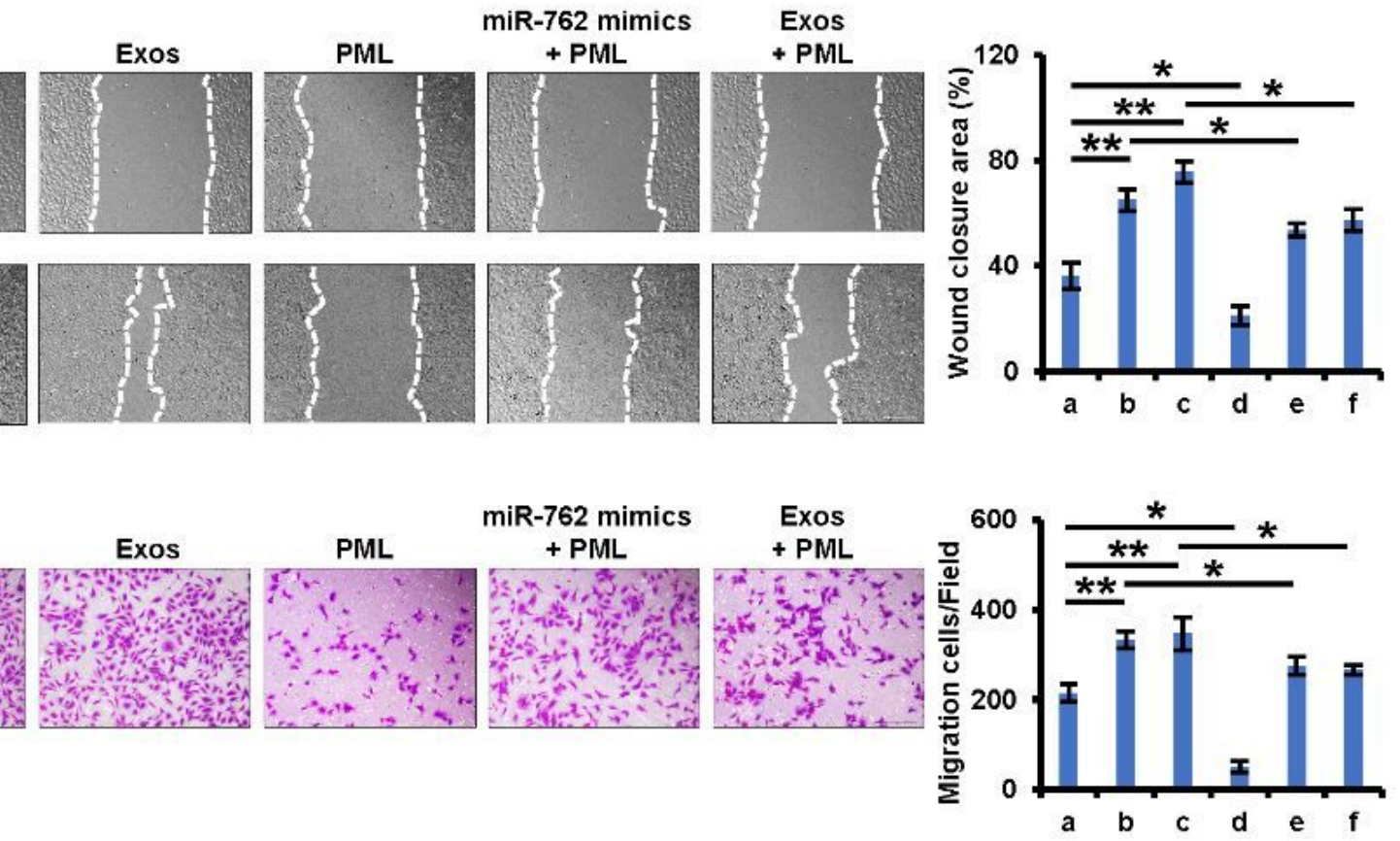

Figure 5 
miR-762 and iPSCs-KCs-Exos promote migration of keratinocyte and endothelial cells by targeting PML. A Predicted miR-762 target sequences in PML 3'-UTR and the mutated nucleotides in the 3'UTR of PML. B The relative luciferase activity in 293T cells with psiCHECK-PML-wt-3'UTR (WT) or psiCHECK-PML-mut3'UTR (MUT) and miR-762 mimics co-transfection was evaluated by luciferase reporter assay. C, D qRTPCR and Western blot analysis of PML expression in HaCaT cells and HUVECs which were treated with control, miR-762 mimics and iPSCs-KCs-Exos (Exos). E Representative images of the wound healing assay and quantitative analysis of the wound healing rates of HaCaT cells, which were treated with a control, b miR-762 mimics, c iPSCs-KCs-Exos (Exos), d pcDNA3.1-PML (PML), e miR-762 mimic plus pcDNA3.1-PML (PML), f iPSCs-KCs-Exos (Exos) plus pcDNA3.1-PML (PML). Scale bar, $200 \mu \mathrm{m}$. F Images of migrated HUVECs and quantitative analysis of the migrated cells, which were treated with $\mathbf{a}$ control, $\mathbf{b}$ miR-762 mimics, c iPSCs-KCs-Exos (Exos), d pcDNA3.1-PML (PML), e miR-762 mimic plus pcDNA3.1PML (PML), f iPSCs-KCs-Exos (Exos) plus pcDNA3.1-PML (PML). Scale bar, $200 \mu \mathrm{m} .{ }^{\star} P<0.05,{ }^{*} P<0.01$.

\section{Figure 6}

PML regulated ITGB1 is involved in the promotion of keratinocytes and endothelial cell migration by iPSCs-KCs-Exos. A, B qRT-PCR and Western blot analysis of ITGB1 expression in HaCaT cells and HUVECs which were treated with control siRNA or PML siRNA. B Western blot analysis of protein expressin of ITGB1 each group. C, D qRT-PCR and Western blot analysis of of ITGB1 expression in HaCaT cells and HUVECs which were treated with control, miR-762 mimics, iPSCs-KCs-Exos. E Representative images of the wound healing assay and quantitative analysis of the wound healing rates of HaCaT cells, which were treated with a control, b miR-762 mimics, c iPSCs-KCs-Exos (Exos), d pcDNA3.1-ITGB1 (ITGB1), e miR-762 mimic plus pcDNA3.1- ITGB1 (ITGB1), f iPSCs-KCs-Exos (Exos) plus pcDNA3.1- ITGB1 (ITGB1). Scale bar, $200 \mu \mathrm{m}$. F Images of migrated HUVECs and quantitative analysis of the migrated cells, which were treated with $\mathbf{a}$ control, b miR-762 mimics, $\mathbf{c}$ iPSCs-KCs-Exos (Exos), d pcDNA3.1-ITGB1 (ITGB1), e miR-762 mimic plus pcDNA3.1- ITGB1 (ITGB1), f iPSCs-KCs-Exos (Exos) plus pcDNA3.1- ITGB1 (ITGB1). Scale bar, $200 \mu \mathrm{m} .{ }^{\star} P<0.05,{ }^{* \star} P<0.01$.

\section{Supplementary Files}

This is a list of supplementary files associated with this preprint. Click to download.

- Agraphicalabstractimage.jpg 